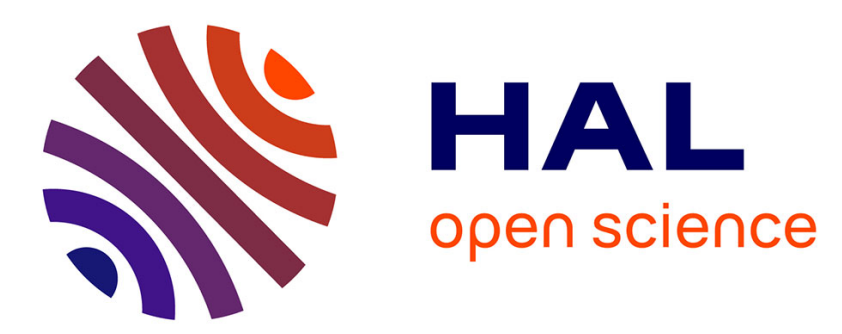

\title{
Estimating Missing Information by Cluster Analysis and Normalized Convolution
}

Davide Guastella, Cesare Valenti

\section{To cite this version:}

Davide Guastella, Cesare Valenti. Estimating Missing Information by Cluster Analysis and Normalized Convolution. IEEE 4th International Forum on Research and Technology for Society and Industry (RTSI 2018), Sep 2018, Palermo, Italy. 10.1109/RTSI.2018.8548454 . hal-02968412

\section{HAL Id: hal-02968412 https://hal.science/hal-02968412}

Submitted on 16 Oct 2020

HAL is a multi-disciplinary open access archive for the deposit and dissemination of scientific research documents, whether they are published or not. The documents may come from teaching and research institutions in France or abroad, or from public or private research centers.
L'archive ouverte pluridisciplinaire HAL, est destinée au dépôt et à la diffusion de documents scientifiques de niveau recherche, publiés ou non, émanant des établissements d'enseignement et de recherche français ou étrangers, des laboratoires publics ou privés. 


\section{Estimating Missing Information by Cluster Analysis and Normalized Convolution}

\author{
Davide Andrea Guastella \\ Institut de Recherche en Informatique de Toulouse \\ Université Toulouse III Paul Sabatier, France \\ davide.guastella@irit.fr
}

\author{
Cesare Valenti \\ Dipartimento di Matematica e Informatica \\ Università degli Studi di Palermo, Italy \\ cesare.valenti@unipa.it
}

\begin{abstract}
Smart city deals with the improvement of their citizens' quality of life. Numerous ad-hoc sensors need to be deployed to know humans' activities as well as the conditions in which these actions take place. Even if these sensors are cheaper and cheaper, their installation and maintenance cost increases rapidly with their number. We propose a methodology to limit the number of sensors to deploy by using a standard clustering technique and the normalized convolution to estimate environmental information whereas sensors are actually missing. In spite of its simplicity, our methodology lets us provide accurate assesses.
\end{abstract}

\section{INTRODUCTION}

The increasing demand for better public services offered by modern cities requires ad hoc support for technologies, all tied together into the so-called smart city concept, that embraces different multidisciplinary fields as informatics, urban and social sciences, politics as well as economics. Nonetheless, the concept of a smart city remains strongly ambiguous; it has been growing from empirical experience and lacks a systemic theoretical study [1].

A study commissioned by the European Union defines smart city initiatives as multi-stakeholder municipally based partnerships aimed at addressing problems of common interest with the aid of information and communications technologies, which underpin the smart classification [2]. Dameri [1] analyzed five areas of study concerning the smart city: intelligent city, digital city, sustainable city, technocity and well-being city. Technocity concerns mainly efficiency and effectiveness improvements of the infrastructures, whereas digital city refers to the representation of several aspects of a real city. These two aspects are not of primary interest for this paper, which will focus on the following definitions:

- an intelligent city is able to produce knowledge and to translate it into unique and distinctive abilities; this city is smart because it is able to create intellectual capital and to ground development and well-being on this intellectual capital;

- a sustainable city aims to become a "green city" by using technology to reduce pollution, to produce energy and to improve the buildings efficiency;

- a well-being city produces the best quality of life for citizens, but also to create regional attractiveness both for people and for business. Technology is just part of the means used to reach these goals, but also culture, climate, history and monuments are considered as important success factors.

These concepts do not represent disjoint areas of analysis and still share some common aspects. Moreover, a system to support a smart city (in terms of both hardware and software) must satisfy the following conditions:

- openness: the system must be able to work with intermittent devices. For example, if we consider devices such as the smart-phones, there is no guarantee that these are always available;

- heterogeneity: observations from heterogeneous devices usually produce large volumes of data that must be pruned and correlated in order to generate a valuable knowledge;

- large-scale: due to the amount of (physical and virtual) entities involved in smart cities and the huge amount of data to process, there is a need for efficient data storing and manipulation techniques. Moreover the data must be always available to the final user;

- unpredictability: it concerns robust systems able to continuously self-adapt to changes that may occur in a highly dynamic environment;

- privacy: non-intrusiveness is considered a key point when collecting data in a smart city.

All these challenges must be addressed when the mobility of ubiquitous and heterogeneous devices introduce different accessibility scenarios with a high level of complexity. Ambient systems represent a key solution to integrate different technologies and provide an IoT infrastructure to be aware of the environment, doing pervasive computing and profiling through human-centric computer interactions [3].

Wang et al. [4] suggested a multi-agent check system where a heuristic intelligent optimization is used to achieve a high level of comfort (with respect to temperature, illumination and the $\mathrm{CO}_{2}$ concentration) with the minimum power consumption.

Costantini et al. [5] defined a multi-agent system approach to control cultural sites such as museum or archaeological sites and to disseminate personalized information to users according to their location.

Ballatore et al. [6] realized a system for querying the 
buildings in a campus to send users information such as room and equipment availability or scheduled classes in courses.

Saldaña-Pérez et al. [7] described a model to provide semantic instructions to move around places through geospatial analysis, semantic processing and mobile technologies. The route starts from the mobile position and proposes points of interest and visual aids along the road.

As a matter of fact, today there is a growing interest to support technologies to support not only smart cities, but also smart regions [8]. Moreover, the deployment of applications in large scale environments is a difficult task due to a variety of constraints (e.g. energy, communication, computing capacities, mobility, autonomy) [9], [10]. Smart tourism supports systems that bind together technologies and systems in different cities to provide specific services to the final user. The method developed by Rivera et al. [11] makes use of preferences, like recommended points of interests and opinions by other users (obtained from the web 2.0 by means of social media), activities declared during vacations, the maximum distance the user is able to walk to provide better touristic itineraries.

Sabatucci et al. [12] developed a smart travel system that acts as a tour operator, combining travel components ondemand to create a holiday package that takes into account user preferences such as budget availability, places of interest and activities to perform.

Among the variety of methods to complete missing information or to handle sparse data, classical methods based on regression analysis use different coefficients for each missing data and take into account a fixed number of data [13], [14]. The model predictive control is based on a mathematical model that describes the environment and it is used to make predictions in smart buildings to reduce their energy consumption while ensuring the occupants' comfort at the same time [15]. Another approach, based on artificial neural networks, was proposed for missing environmental information [16], although it needs to specify a precise architecture as well as the activation function. These parameters greatly depend on the case study. Vice versa, normalized convolution is an efficient approach that requires very low computational power and requires just a few parameters that can be pre-defined easily.

This paper focuses on a simple yet effective methodology to estimate missing environmental information by cluster analysis and normalized convolution (see fig. 1). We verified the proposed approach on a real weather dataset, freely available on the Internet, showing good results for applications on a regional scale. The paper is organized as follows: section II faces the problem of clustering by using a hierarchical approach together with deciding automatically the optimal number of clusters; section III describes the normalized convolution technique, used the extrapolate missing information; section IV reports the experimental setup, enhances the hierarchical clustering by means of the Voronoi diagram and discusses the final results; section $\mathrm{V}$ summarizes the applicability of the proposed methodology and describes research perspectives.

\section{Agglomerative hierarchicAl Clustering}

In a clustering problem we are given a dataset of "points" and the goal is to partition this dataset into a finite set of disjoint subsets (i.e. clusters) $C$ such that the union of all subsets covers the whole dataset. A high quality clustering is one in which the points in any particular subset are more similar to each other than the points in other subsets [17].

One common technique for clustering is known as agglomerative hierarchical clustering; this approach starts considering each single point into a single cluster and then it iteratively merges the closest pair of clusters according to some similarity criteria until all points belong to one cluster. The main drawbacks of this approach are that the points that have been incorrectly grouped at an early stage cannot be reallocated subsequently and different similarity measures among the clusters can lead to completely different results [18].

The hierarchical methods group training points into a typical tree structure known as dendrogram which represents a sequence of nested cluster constructed top-down or bottomup. The root of the tree represents the cluster that includes the whole dataset of points while each leave has one point. By cutting the tree at a certain level we obtain a clustering into disjoint groups (fig. 2) [19].

A major challenge in cluster analysis is determining the optimal number of clusters. There are two principal approaches to face this problem. One common technique depends on plotting an optimization parameter against a number of clusters and on choosing the number which corresponds to a large change in the parameter value. A second method seeks large changes in inter-group fusions where fusion distances are generally determined with the aid of the dendrogram. Both these techniques depend on the determination of relatively large changes in an index rather than its minimization or maximization and therefore require human interpretation and subjective analysis of what should be considered a "large change" [20]. In the gap statistic method [21] we suppose that we clustered the set of points into $k$ clusters $C_{1}, C_{2}, \ldots, C_{k}$. Let

$$
D_{n}=\sum_{i, j \in C_{n}} d_{i j}
$$

be the sum of pairwise distances for all points in $C_{n}$ and let

$$
W_{k}=\sum_{n=1}^{k} \frac{D_{n}}{2\left\|C_{n}\right\|}
$$

be the variance that represents be the dispersion of the points within the $k$ clusters. The idea of gap statistic is to compare the dispersion of the points within each of the $k$ clusters to its expectation under an appropriate null reference distribution [21].

\section{Normalized CONVOLUtion}

Dealing with irregularly sampled data due to the presence, for example, of noise or instrumental error is quite common in many scientific fields, such as astrophysics, geoscience, 


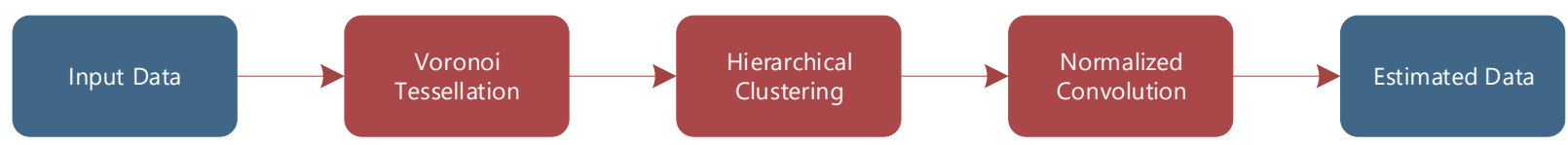

Fig. 1. Sketch of the main steps of our methodology.

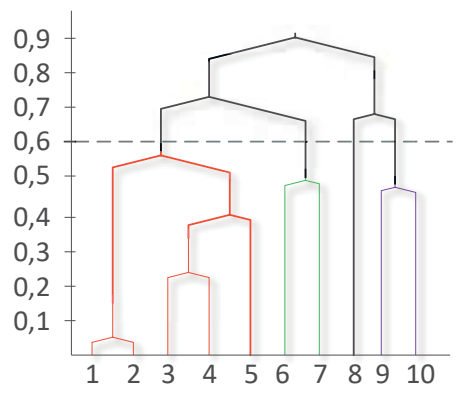

Fig. 2. Colors represents the clusters obtained by thresholding the dendrogram at 0.6 (the dashed line).

oceanography, telecommunications, remote sensing and medical imaging. In general, performing operations on incomplete or irregularly sampled data is a non-trivial task and therefore it is often required to reconstruct the irregularly sampled data or resample it onto a regular grid. These operations can be carried out through direct methods, which can involve the computation of irregularly sampled data in the frequency domain and the inverse transform to obtain a regularly spaced signal [22], [23], [24]. Normalized convolution is a non-direct methodology widely used for filtering incomplete or uncertain data which is based on the separation of both data and operator into a signal part and a certainty part. A map indicates the presence degree of a sample in a given position and, in particular, a binary map would indicate just the absence or presence of the signal. This approach was described for the first time by Knutsson and Westin for digital image analysis with a simple and fast implementation [25].

Let $S$ be the positive map that represents certain samples of bi-dimensional data $I$. If we indicate by $\{S \cdot I\}$ the pointwise product of $S$ and $I$ and by $\{K * I\}$ the usual convolution with a kernel $K$, then normalized convolution is defined by

$$
N C(I, S, K)=\{K * S \cdot I\} /\{K * S\}
$$

In other words, to reconstruct the data $I$ from its samples specified in $S$, we just have to weight $\{K * S \cdot I\}$ by the confidence $\{K * S\}$ of the results generated.

The kernel, centered in the origin, have the original and general form

$$
K_{x, y}= \begin{cases}r^{-\alpha} \cos ^{\beta}\left(\frac{\pi r}{2 R}\right) & \text { if } r<R \\ 0 & \text { otherwise }\end{cases}
$$

where $r=\sqrt{\left(x^{2}+y^{2}\right)}$. Usually, the parameters $\alpha=0$ and $\beta=2$ are used, thus obtaining the bi-dimensional raised cosine depicted in figure 3 .
To avoid over-smoothing the output signal, $K$ should be big enough to contain just some pixels of the input signal. Vice versa, if the distance between the nearest samples in $S$ is greater than the size $R$ of $K$, then the reconstructed image will contain gaps. Without a priori information, $R$ is automatically set to the minimal distance among the available samples to reduce artifact effects along discontinuities: at least one point lies always within the radius [26].

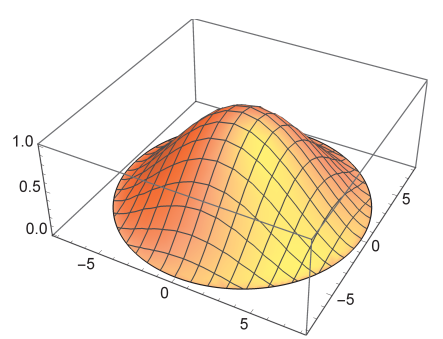

Fig. 3. Graphic representation of the typical smoothing kernel, as described in $[25]$.

It is noteworthy that the normalized convolution can be calculated through efficient lookup tables if we accumulate the contribution of the few known samples according to the reflected kernel's values about its center (fig. 4), instead of directly calculating the scalar product at each unknown point. Instead, this latter approach is preferable if we are interested in a single missing point, without considering the rest of the signal (fig. 5).

A variant of this algorithm is known as adaptive normalized convolution and it modulates both the size and the shape of the kernel $K$, according to the position of certain samples [27], [28]. In this case, implementing an optimized and efficient custom convolution routine can be quite difficult: a different filter should be arranged for each point of the output image and an estimate of the gradient of the whole signal is used to determine this proper kernel. Obviously, this gradient itself is just an approximation since it has to be computed from available samples specified by $S$. Actually, we preferred not to use this approach because it requires a considerable amount of computational time and usually its performances does not justify the enhancement of the final result due to the very few known samples.

\section{MATERIALS AND METHOD}

To evaluate the correctness of the proposed methodology we considered the freely available temperatures in degree Celsius recorded by Arpae-SIMC, a weather service of the EmiliaRomagna region in Italy which provides weather warnings to the Italian Civil Protection Department. Beyond climatological 


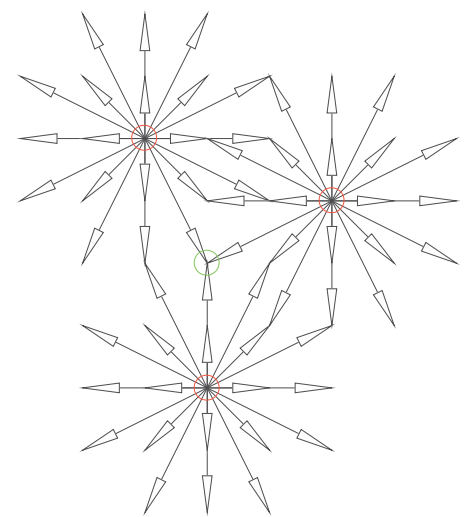

Fig. 4. If very few sensors (marked in red) are available, it is convenient to calculate the values in all remaining points by indirect contributions of the actual temperatures: their precalculated products by the kernel are simply added to accumulation arrays.

Fig. 5. If we are interested in estimating the temperature in just one point (marked in green), the straightforward implementation of the convolution remains the fastest solution.

data, Arpae-SIMC provides tools, products and indicators to evaluate the impact of storms on the coast [29].

About $48 \%$ of this region consists of plains while $27 \%$ is hilly and $25 \%$ is mountainous. We considered the average daily air temperatures at 2 meters of altitude, collected in 196 days from September 82017 to April 252018 by 80 weather stations. Actually, since we do not make a temporal analysis, we have not considered the days when some stations were not operational between those two dates. That is, the minimal form of this dataset consists in an array of $196 \times 80$ numerical values. The exact geographic locations of these stations were corrected to the Cartesian plane through the Mercator projection [30].

The main steps of the proposed methodology are depicted in figure 1. At first, a Voronoi tessellation [31] is calculated according to the actual positions of the stations: this delimits their rough relevance areas (fig. $6 a$ ). Given a station, its Voronoi tile is a convex polygon that includes all points closer to that station than to any other station. This way, each tile contains exactly one station. The Voronoi tessellation can be calculated efficiently by mathematical morphology [32].

Actually, some stations (especially if close to each other) can show similar detected temperature; a hierarchical clustering technique groups together these stations and therefore their corresponding tiles. The hierarchical clustering tree is created starting from the information $I$ of the dataset and then the optimal number $k$ of clusters is computed by using the gap statistic method. Stations belonging to the same cluster and in adjacent Voronoi tiles are grouped together: this refines the original clustering by separating eventual stations which act in the same manner though are too far one from each other (fig. 6b). These new tiles are no more convex in general and contain stations with a similar behavior, normally due to the local orography.

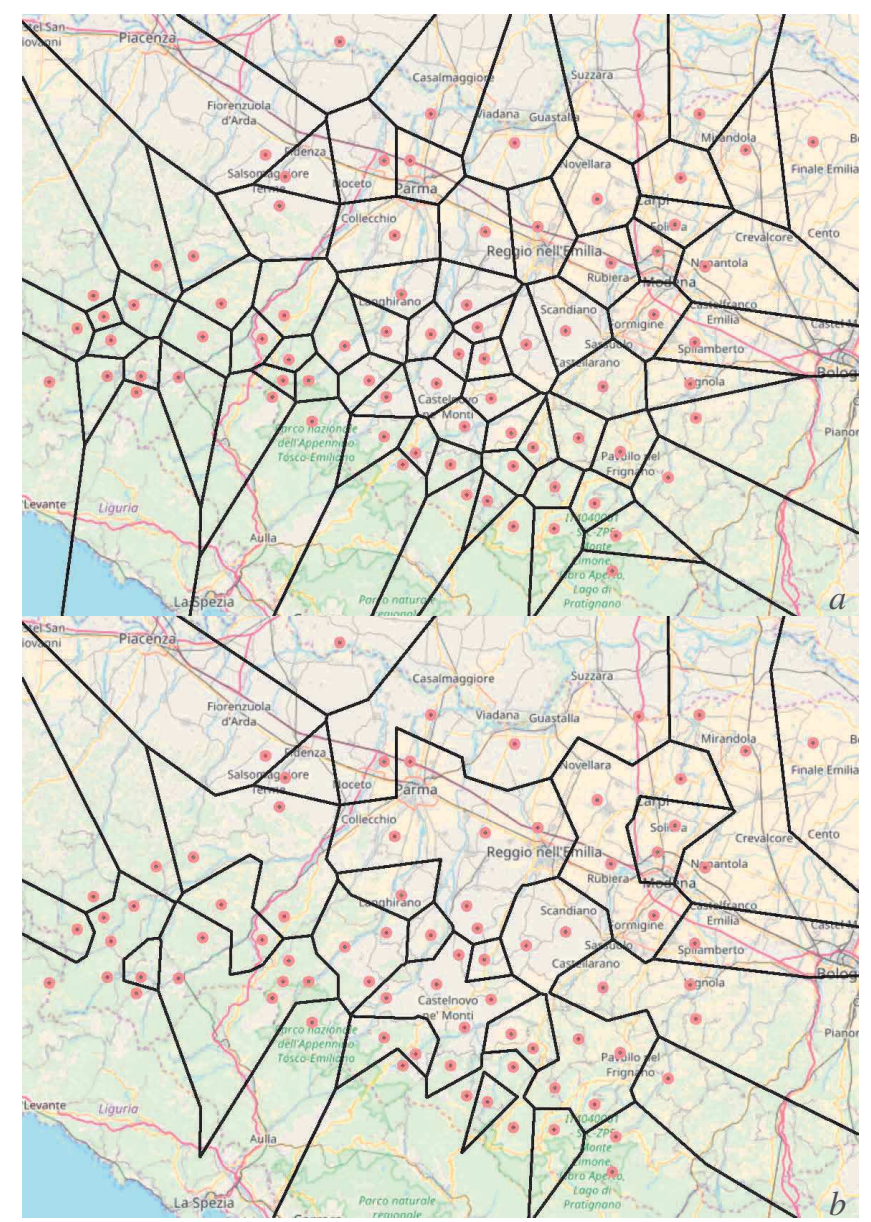

Fig. 6. Superimposition of the Voronoi diagram (a) and subsequen clusters $(b)$ on the map of Emilia-Romagna (obtained through OpenStreetMap [33]). Red dots indicate the weather stations.

The certainty map $S$ needed by the normalized convolution stores just the positions of the stations. In order to estimate the temperature value in a missing position, it is sufficient to apply the normalized convolution on the information map $I$ from the dataset and on the certainty map $S$, limited to the cluster containing the position (fig. 7).

All experiments were carried out on an entry level machine, equipped with i7-7820HQ, 32GB RAM and Windows 10. Our algorithm was coded in Matlab language without particular optimizations; nonetheless it takes about a second to cluster the whole dataset and it is practically instantaneous in computing the normalized convolution. It should be noted that the clusters should be computed from time to time and while it is appropriate for a server to update the cluster periodically, the normalized convolution can be efficiently calculated even by low-end mobile devices. That is, the certainty map $S$ can 


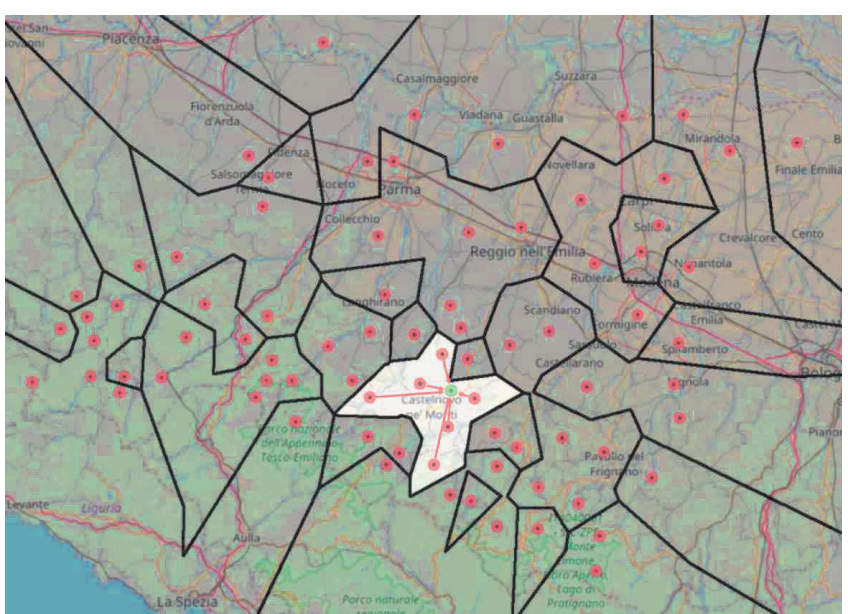

Fig. 7. Only the stations within the same cluster (in detail) contribute to the normalized convolution, similarly to figure 5 .

vary not only according to the response provided by the fixed stations, but it can be refined also through extemporaneous surveys.

To verify the correctness of the results we considered a leave-one-out cross validation: for each experiment a precise station has been removed at a time, in order to evaluate the estimation from the remaining stations. Figure 8 shows the average error and the standard deviation of each station during the considered 196 days. The average overall error is just $-0.03^{\circ}$.

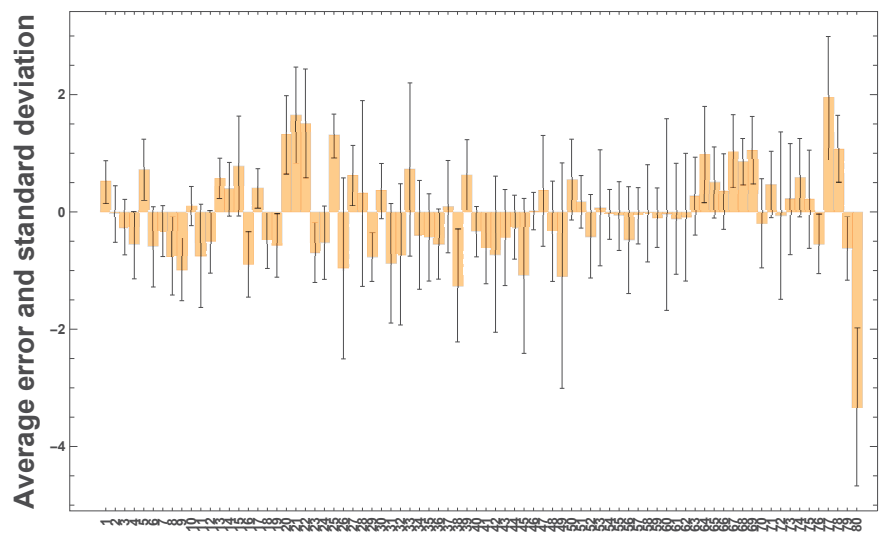

Weather station

Fig. 8. Error bar of deduced temperatures (degree Celsius) for each station, computed through the leave-one-out process.

The same normalized convolution can be effectively applied to determine the reliability of the proposed method on the entire lattice: it is sufficient to propagate the average error of each weather station to measure the correctness of the extrapolated measurements in any point without a real station (fig. 9).

\section{Conclusion And Perspectives}

This paper introduces a simple and effective non-supervised methodology for estimating missing environmental information in a large scale context through cluster analysis and

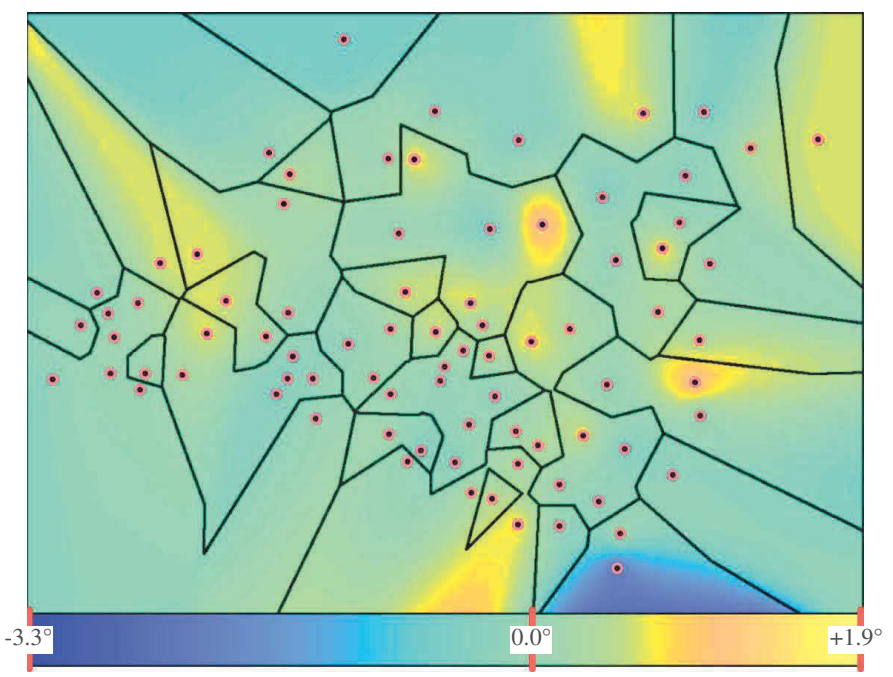

Fig. 9. Expected error through normalized convolution. Most stations do not introduce any error (green); some stations report warmer values than the real temperatures (yellow); only one station indicates a lower temperature (blue). We highlight that this colder station (\#80 in fig. 8) is due to the absence of neighboring stations for a correct evaluation.

normalized convolution. We believe the method could be further improved: for example, we considered a standard off-line hierarchical clustering algorithm while on-line techniques [34] could update dynamically the clustering tree each time the system receives new information (e.g. mobile devices equipped with proper sensors). Also geographical characteristics of the stations such as altitude can be used to provide a better grouping in the Voronoi diagram.

The proposed methodology is scale-independent, since it makes no assumption on the spatial distribution of the stations. Moreover, it is applicable to a variety of sensors and it does not require any intervention by the user. Although we considered only temperatures from a real regional dataset, the methodology is general enough to be applied to a variety of environments. Indeed, we plan to use the same approach to determine technologies that could be employed in the smart-campus context of the University of Toulouse III Paul Sabatier [35]. As a matter of facts, more and more researchers consider universities as great places to experiment innovative services and techniques for smart buildings. Installing and maintaining a large number of different sensors to monitor all parameters of the campus is quite expensive.

According to the quantitative results, our methodology already provides satisfactory temperature estimations by considering a reduced subset of weather stations, while the clustering step is suggested from time to time to process the whole dataset. To improve the performances it would be possible to cluster data streams by accessing the input information via a few linear scans; these algorithms do not store large amount of data by processing only a very limited subset of data [36]. This idea is due to emerging smart applications which involve massive data such as traffic, services and environmental data. We plan to investigate in our future works different techniques 
to produce missing information, in order to carry out a comparative analysis.

\section{ACKNOWLEDGEMENT}

This work was supported partially by the neOCampus initiative (neocampus.org) from the University Toulouse III Paul Sabatier [35].

\section{REFERENCES}

[1] R. Dameri, "Searching for Smart City definition: a comprehensive proposal," International Journal of Computers \& Technology, vol. 11, no. 5, pp. 2544-2551, 2013.

[2] C. Manville, G. Cochrane, J. Cave, J. Millard, J. Pederson, R. Thaarup, A. Liebe, M. Wissner, R. Massink, and B. Kotterink, "Mapping Smart Cities in the EU," tech. rep., European Parliament, 2014.

[3] J. Viterbo, L. Mazuel, Y. Charif, M. Endler, N. Sabouret, K. Breitman, A. El Fallah Seghrouchni, and J.-P. Briot, "Ambient intelligence: Management of distributed and heterogeneous context knowledge," CRC Studies in Informatics Series. Chapman \& Hall, pp. 1-44, 2008.

[4] Z. Wang, L. Wang, A. Dounis, and R. Yang, "Multi-agent control system with information fusion based comfort model for smart buildings,' Applied Energy, vol. 99, pp. 247-254, 2012.

[5] S. Costantini, L. Mostarda, A. Tocchio, and P. Tsintza, "DALICA: Agent-Based Ambient Intelligence for Cultural-Heritage Scenarios," IEEE Intelligent Systems, vol. 23, no. 2, pp. 34-41, 2008.

[6] A. Ballatore, T. Pham, J. Yin, L. Truong-Hong, and J. Carswell, "Design and development of personal geoservices for universities," in International Workshop on Information Fusion and Geographic Information Systems: Deep Virtualization for Mobile GIS, vol. 216 of Lecture Notes in Geoinformation and Cartography, pp. 3-26, Springer, 2015.

[7] A. Saldaña-Pérez, M. Torres-Ruiz, M. Moreno-Ibarra, and G. GuzmánLugo, "RRMA Referenced Routing Model to Generate a Semantic Service of Navigation in Mobile Devices," in Information Fusion and Geographic Information Systems, pp. 43-58, Springer, 2015.

[8] J. Vuppalapati, S. Kedari, A. Ilapakurthy, A. Ilapakurti, and C. Vuppalapati, "Smart dairies-enablement of smart city at gross root level," in International Conference on Big Data Computing Service and Applications, pp. 118-123, IEEE, 2017.

[9] A. Olaru, A. Florea, and A. El Fallah Seghrouchni, "A Context-Aware Multi-Agent System as a Middleware for Ambient Intelligence," Mobile Networks and Applications, vol. 18, no. 3, pp. 429-443, 2013.

[10] F. Piette, C. Dinont, A. El Fallah Seghrouchni, and P. Taillibert, "Deployment and configuration of applications for ambient systems," in International Conference on Ambient Systems, vol. 52 of Procedia Computer Science, pp. 373-380, Elsevier, 2015.

[11] L. Cabrera Rivera, L. Vilches-Blzquez, M. Torres-Ruiz, and M. Moreno Ibarra, "Semantic recommender system for touristic context based on linked data," in International Workshop on Information Fusion and Geographic Information Systems: Deep Virtualization for Mobile GIS, vol. 216 of Lecture Notes in Geoinformation and Cartography, pp. 77-89, Springer, 2015.

[12] L. Sabatucci, A. Cavaleri, and M. Cossentino, "Adopting a middleware for self-adaptation in the development of a smart travel system," in International Conference on Intelligent Interactive Multimedia Systems and Services, vol. 55 of Smart Innovation, Systems and Technologies, pp. 671-681, Springer, 2016.

[13] Y. Xia, P. Fabian, A. Stohl, and M. Winterhalter, "Forest climatology: estimation of missing values for bavaria, germany," Agricultural and Forest Meteorology, Elsevier, vol. 96, no. 1, pp. 131-144, 1999.

[14] G. T. Ferrari and V. Ozaki, "Missing data imputation of climate datasets: Implications to modeling extreme drought events," Revista Brasileira de Meteorologia, Scielo, vol. 29, pp. 21-28, 032014.

[15] F. Oldewurtel, A. Parisio, C. N. Jones, D. Gyalistras, M. Gwerder, V. Stauch, B. Lehmann, and M. Morari, "Use of model predictive control and weather forecasts for energy efficient building climate control,' Energy and Buildings, Elsevier, vol. 45, pp. 15-27, 2012.
[16] S. S. Zanetti, E. F. Sousa, V. P. Oliveira, F. T. Almeida, and S. Bernardo, "Estimating evapotranspiration using artificial neural network and minimum climatological data," Journal of Irrigation and Drainage Engineering, ASCE, vol. 133, no 2, pp. 83-89, 2007.

[17] A. Kobren, N. Monath, A. Krishnamurthy, and A. McCallum, "A hierarchical algorithm for extreme clustering," in International Conference on Knowledge Discovery and Data Mining, pp. 255-264, ACM, 2017.

[18] G. Gan, C. Ma, and J. Wu, Data Clustering: Theory, Algorithms and Applications. Statistics and Applied Probability, SIAM, 2007.

[19] M. Rafsanjani, Z. Varzaneh, and N. Chukanlo, "A Survey Of Hierarchical Clustering Algorithms," Journal of Mathematics and Computer Science, vol. 5, no. 3, pp. 229-240, 2012.

[20] D. Davies and D. Bouldin, "A Cluster Separation Measure," IEEE Trans. Pattern Anal. Mach. Intell., vol. 1, no. 2, pp. 224-227, 1979.

[21] R. Tibshirani, G. Walther, and T. Hastie, "Estimating the number of clusters in a data set via the gap statistic," Journal of the Royal Statistical Society: Series B (Statistical Methodology), vol. 63, no. 2, pp. 411-423, 2001.

[22] F. Marvasti, Nonuniform Sampling. Theory and Practice. Information Technology: Transmission, Processing and Storage, Kluwer Academic/Plenum Publishers, 2001.

[23] L. Greengard and J.-Y. Lee, "Accelerating the Nonuniform Fast Fourier Transform," SIAM Review, vol. 46, no. 3, pp. 443-454, 2004.

[24] X. Chen, J.-H. Jung, and A. Gelb, "Finite Fourier Frame Approximation Using the Inverse Polynomial Reconstruction Method," Journal of Scientific Computing, Springer, pp. 1-21, 2018.

[25] H. Knutsson and C.-F. Westin, "Normalized and differential convolution," in Computer Vision and Pattern Recognition, pp. 515-523, IEEE, 1993.

[26] G. Albanese, M. Cipolla, and C. Valenti, "Genetic Normalized Convolution," in International Conference on Image Analysis and Processing, vol. 6978 of Lecture Notes in Computer Science, pp. 670-679, Springer, 2011.

[27] T. Pham and L. van Vliet, "Normalized Averaging Using Adaptive Applicability Functions with Applications in Image Reconstruction from Sparsely and Randomly Sampled Data," in Image Analysis, pp. 485-492, Springer, 2003.

[28] T. Pham, L. van Vliet, and K. Schutte, "Robust Fusion of Irregularly Sampled Data Using Adaptive Normalized Convolution," Journal on Advances in Signal Processing, pp. 1-12, 2006.

[29] L. Bressan, A. Valentini, T. Paccagnella, A. Montani, C. Marsigli, and M. Tesini, "Sensitivity of sea-level forecasting to the horizontal resolution and sea surface forcing for different configurations of an oceanographic model of the Adriatic Sea," Advances in Science and Research, vol. 14, pp. 77-84, 2017.

[30] M. Monmonier, Rhumb lines and map wars: a social history of the Mercator projection. University of Chicago Press, 2010.

[31] A. Okabe, B. Boots, K. Sugihara, and S. Chiu, Spatial Tessellations Concepts and Applications of Voronoi Diagrams. Probability and Statistics, John Wiley, 2000

[32] D. Guastella and C. Valenti, "Cartoon filter via adaptive abstraction," Journal of Visual Communication and Image Representation, vol. 36, pp. 149-158, 2016.

[33] OpenStreetMap contributors, "Planet dump retrieved from https://planet.osm.org." https://www.openstreetmap.org, 2017.

[34] R. Pensa, D. Ienco, and R. Meo, "Hierarchical co-clustering: off-line and incremental approaches," Data Mining and Knowledge Discovery, Springer, vol. 28, no. 1, pp. 31-64, 2014.

[35] M.-P. Gleizes, J. Boes, B. Lartigue, and F. Thibolt, neOCampus: Demonstrator of Connected, Innovative, Intelligent and Sustainable Campus, vol. 76, pp. 482-491. Springer, 2018.

[36] S. Guha and N. Mishra, "Clustering data streams," in Data Stream Management: Processing High-Speed Data Streams, pp. 169-187, Springer, 2016. 